\title{
НАРУШЕНИЯ РИТМА И ПРОВОДИМОСТИ СЕРДЦА У ПАЦИЕНТОВ С АКРОМЕГАЛИЕЙ: ВСТРЕЧАЕМОСТЬ, ВЛИЯНИЕ ДЛИТЕЛЬНОСТИ ЗАБОЛЕВАНИЯ, ЛЕКАРСТВЕННОЙ ТЕРАПИИ И ФИБРОЗА МИОКАРДА
}

\author{
Мелкозеров К.В., Пржиялковская Е.Г., Тарбаева Н.В., Алмасханова А.А., Куклина М.Д., Алферова П.А., \\ Гомова И.С., Белоусов Л.А., Калашников В.Ю., Белая Ж.Е., Мокрышева Н.Г. \\ ФГБУ «НМИЦ эндокринологии» Минздрава России, Москва
}

АКТУАЛЬНОСТЬ: сердечно-сосудистые осложнения, включая нарушение ритма и проводимости сердца (НПРС) составляют более $60 \%$ от всех летальных исходов пациентов с акромегалией. НРПС выявляются у 40\% больных акромегалий и могут приводить к внезапной сердечной смерти. Следствием гиперсекреции соматотропного гормона и инсулиноподобного фактора роста-1 является формирование гипертрофической кардиомиопатии и миокардиального интерстициального фиброза. В результате чего, измененная структура миокарда приводит к электрофизиологическим нарушениям. Дополнительным фактором риска развития НРПС может являться терапия аналогами соматостатина, используемая для лечения акромегалии.

Несмотря на достижение целевых значений СТГ и ИФР-1 на фоне лечения, возникшие осложнения акромегалии часто являются необратимыми. Поэтому необходимо выявление группы пациентов с акромегалией, имеющих высокий риск развития жизнеугрожающих НРПС для своевременного лечения и профилактики ВСС, а также повышения качества жизни данных пациентов.

ЦЕЛЬ: оценить частоту и тяжесть НРПС, выявить факторы риска, способствующие развитию НРПС у больных акромегалией, встречаемость фиброза миокарда по данным магнитно-резонансной томографии (MPT) сердца у пациентов С НРПС.

МАТЕРИАЛЫ И МЕТОДЫ: проведено одноцентровое одномоментное исследование, в которое включен 461 пациент (151 мужчина и 310 женщин) с акромегалией, госпитализированные в ФГБУ «НМИЦ эндокринологии» Минздрава России в период с января 2018г. по март 2020г. Всем пациентам выполнено стандартное медицинское обследование, включающее гормональное исследование крови, регистрацию электрокардиограммы, эхокардиографию, холтеровское мониторирование ЭКГ. МРТ сердца с контрастированием гадолинием выполнено 18 пациентам с НРПС (11 мужчин и 7 женщин).

РЕЗУЛЬтАТЫ: результаты нашего исследования демонстрируют высокую встречаемость НРПС у пациентов с акромегалией - 42\%. Наиболее частыми видами НРПС были синусовая брадикардия (СБ) - 19,1\% случаев и нарушение проводимости по ножкам пучка Гиса - 14,5\%. Между тем, СБ обычно наблюдались у пациентов, которые лечились аналогами соматостатина, по сравнению с пациентами, которые не получали данную терапию (50\% и 38,6\% соответственно, $p=0,004)$. Однако урежение пульса менее 50 в минуту наблюдалось только у $27,3 \%$. Несмотря на то, что терапия аналогами соматостатина увеличивала риск развития СБ, только 41,6\% пациентов в группе с ЧСС менее 50 в минуту получали данный вид лечения. Мужчины чаще страдали НРПС, чем женщины (54,2\% и 37,4\% соответственно, $\mathrm{p}=0,0005)$. Выявлено, что развитие НРПС зависит, в первую очередь от длительности акромегалии ( $(\mathrm{p}=0,04)$, в то время как активность заболевания не оказывает значимого влияния. По нашим данным пациенты с НРПС имели более низкие показатели ИФР-1 и СТГ в отличие от пациентов без НРПС. 61\% пациентов с акромегалией и НРПС, которым была проведена МРТ сердца, имели признаки фиброза миокарда. В тоже время, признаки фиброза миокарда регистрировались у пациентов при отсутствии значительных отклонений от нормы по данным ЭХО-КГ.

ВЫВОды: в исследовании выявлена высокая встречаемость нарушений ритма и проводимости сердца среди пациентов с акромегалией, которая составила 42\%. НРПС часто наблюдаются у пациентов с акромегалией, несмотря на достижение ремиссии заболевания. Существует риск развития синусовой брадикардии у больных акромегалией, независимо от проведения терапии аналогами соматостатина. Ранняя диагностика кардиромиопатии только при помощи ЭХО-КГ может быть затруднительна. Учитывая высокую встречаемость фиброза миокарда по данным МРТ у больных акромегалией с НРПС, можно предполагать его влияние на развитие аритмий, однако для уточнения этого требуется продолжение исследования.

КОНФЛИКТ ИНТЕРЕСОВ: не заявляется.

КЛЮЧЕВЫЕ СЛОВА: акромегалия; нарушение ритма и проводимости сердиа; магнитно-резонансная томография сердиа; акромегалическая кардиомиопатия; миокардиальный фиброз. 